\title{
The Novel Biomarkers-Based HALP (Hemoglobin, Albumin, Lymphocyte And Platelet)-Prognostic Model For Acute And Subacute Patients With Cerebral Venous Sinus Thrombosis: A Retrospective Cohort Study
}

\section{Shen Li}

Zhengzhou University First Affiliated Hospital

Kai Liu

Zhengzhou University First Affiliated Hospital

\section{Yuan Gao}

Zhengzhou University First Affiliated Hospital

\section{Lu Zhao}

Zhengzhou University First Affiliated Hospital

Hui Fang

Zhengzhou University First Affiliated Hospital

\section{Rui Zhang}

Zhengzhou University First Affiliated Hospital Hongbing Liu

Zhengzhou University First Affiliated Hospital Jiawei Zhao

Zhengzhou University First Affiliated Hospital

\section{Zongping Xia}

Zhengzhou University First Affiliated Hospital

\section{Yuming Xu}

Zhengzhou University First Affiliated Hospital

\section{Bo Song ( $\nabla$ songbo76@sina.com )}

Zhengzhou University First Affiliated Hospital https://orcid.org/0000-0001-9172-1249

\section{Research}

Keywords: Hemoglobin, Albumin, Lymphocyte, and Platelet (HALP), acute and subacute cerebral venous sinus thrombosis, prognostic model 
Posted Date: October 20th, 2020

DOI: https://doi.org/10.21203/rs.3.rs-91558/v1

License: (c) (i) This work is licensed under a Creative Commons Attribution 4.0 International License. Read Full License 


\section{Abstract}

Background: To evaluate the prognosis values of HALP in acute or subacute cerebral venous sinus thrombosis (CVST), to explore the new prognostic model for CVST.

Methods: Consecutive patients who were diagnosed as having acute and subacute CVST were retrospectively investigated. We determined the patients' functional outcomes by modified Rankin Scale (mRS). Multivariate logistic regression analysis was used to assess the relationship between factors and poor functional outcomes. The area under the ROC curve (AUC) was estimated to evaluate the ability of markers and models in predicting clinical prognosis. The prognostic model was presented as nomogram. In addition, the decision curve analysis (DCA) was used to analyze the benefit of this model. Furthermore, survival curves were described by the Kaplan-Meier analysis.

Results: A total of 270 patients were included of which 31 had poor outcome. Multivariable logistic regression analysis demonstrated HALP $(\mathrm{OR}=0.978,95 \% \mathrm{Cl}$ : $0.958-0.999, P=0.039)$ was a protective predictor of outcome. The AUC of HALP was 0.749 (95\% Cl: $0.633-0.865, P=0.044)$. DCA demonstrated that this model significantly improved risk prediction at threshold probabilities of CVST at 0 to $85 \%$. Patients with higher HALP $(P=0.006)$ presented higher overall survival rates.

Conclusions: HALP may be a potential protective marker in acute and subacute CVST patients. The new prognostic model with HALP had potentially better value for Chinese CVST patients.

\section{Highlights}

- HALP values firstly were analyzed in prognosis of stroke, especially in acute and subacute CVST

- Higher HALP value may indicate an independent predictor for good prognosis in patients.

- The novel prognostic model with HALP had potential value for Chinese CVST patients.

\section{Background}

Cerebral Venous Sinus Thrombosis (CVST) is a rare type of stroke, accounting for $0.5-1 \%$ of all strokes ${ }^{1}$. Compared to arterial stroke, CVST has highly variable pathogenic factors and complex clinical symptoms, which lacks of specificity and leads to misdiagnosed ${ }^{2}$. There have been many efforts to find useful prognostic markers and models for monitoring CVST patients, such as platelet to lymphocyte ratio $(\mathrm{PLR})^{3}$, neutrophil to lymphocyte ratio $(\mathrm{NLR})^{4}, \mathrm{C}$ reactive protein (CRP) and D-dimer ${ }^{5}$. Furthermore, the CVST prognostic score based on the International Study on Cerebral Vein and Dural Sinus Thrombosis (ISCVT-RS) ${ }^{6}$ and Cerebral Venous Thrombosis grading scale (CVT-GS) ${ }^{7}$ score were useful models in prognosis of CVST patients. However, some studies have indicated that the scales were not proper for Chinese patients ${ }^{8}$. Therefore, it is necessary to increase external items or explore some new prognosis models which are suitable for more patients. 
The combination of hemoglobin, albumin, lymphocyte and platelet (HALP) was defined as follows: hemoglobin $(\mathrm{g} / \mathrm{L}) \times$ albumin $(\mathrm{g} / \mathrm{L}) \times$ lymphocyte $(/ \mathrm{L}) /$ platelet $(/ \mathrm{L})^{9}$. It was the integration of these four indexes and relatively more stable than single blood parameters. HALP was closely associated with clinical features and was an independent prognostic factor in several types of malignant tumors, including genitourinary cancer, bladder cancer and renal cell carcinoma ${ }^{10-12}$. However, there were no reports concerned with roles of HALP in CVST patients, especially in acute and subacute CVST patients.

In this retrospective study, we aimed to analyze the association between HALP values and prognosis in acute and subacute CVST patients, and to explore the predictive new models for prognosis in these patients.

\section{Methods}

\section{Patient Selection}

Patients included in the retrospective cohort study were from the database of the Henan CVST Registry in the First Affiliated Hospital of Zhengzhou University (Henan, China). All patients diagnosed with CVST from January 2013 through December 2018 were identified. We selected acute/subacute patients from our database. Inclusion criteria were as follows: (1) meeting the diagnostic criteria for CVST established by the American Heart Association/American Stroke Association in $2014^{8}$; (2) filling defect or obstruction of cerebral sinus in the magnetic resonance venogram, digital subtraction angiography, or operation searching; (3) clinical features such as vomiting, visual disturbances, focal neurologic deficit, seizure, and other typical symptoms; (4) acute and subacute patients whose time from onset to addition was less than 30 days $^{13,14}$, or named non-chronic patients; (5) an initial blood sample for laboratory testing 12 hours of admission. Exclusion criteria were as follows: (1) patients with unrelated other serious brain lesions, serious lung disease, or heart disease; (2) patients with undesirable follow-up, including refusal or loss to follow-up; (3) patients less than 18 years old; (4) patients without complete clinical data; (5) chronic patients whose time from onset to addition was more than 30 days. The retrospective cohort study was approved by the Ethics Committee of the First Affiliated Hospital of Zhengzhou University, and patients signed the informed consent form.

\section{Data collection}

Clinical data such as age, gender, clinical presentation, laboratory and imaging tests were collected. Laboratory samples were routinely collected after 12 hours of fasting upon admission to the hospital. The inter-rater reliability for involvement of intracranial venous sinus between two investigators was assessed in some cases.

\section{Evaluation of prognosis}

We evaluated the modified Rankin Scale (mRS) to determine the patients' functional outcomes: $\mathrm{mRS}$ 0-2 as good outcomes, mRS 3-6 as poor outcomes, and death was defined with mRS score of 6 . Follow-up 
information was recorded by telephone interview. Telephone interviewers were not involved in the registry and were blinded to the baseline data. The overall survival time was defined as the date of admission to the date of death from any cause, or to the last follow up date.

\section{Statistical analysis}

All statistical analyses were performed using SPSS 21.0 software. Continuous variables were expressed as mean \pm standard deviation or median, which were analyzed by independent student t-test or MannWhitney test as appropriate. Categorical variables were presented as numbers which were analyzed using Chi-square test or Fisher exact test. The association between these markers and prognosis was explored using multivariate logistic regression analysis. Additionally, we created a heat map to show these results. The area under the ROC curve (AUC) was estimated to evaluate the ability of markers in predicting clinical prognosis. According to the threshold values of HALP, we divided the patients into two groups, including higher HALP group and lower HALP group. Besides, the prognostic model was presented as nomogram for individual patient. Clinical usefulness and net benefit were estimated with decision curve analysis (DCA). Survival curves were described by the Kaplan-Meier analysis and compared with Log-Rank test in higher HALP group and lower HALP group. Two-tailed $P<0.05$ were considered significant.

\section{Results}

We included 297 patients confirmed acute and subacute CVST admitted during the study period from database. We excluded 8 patients because of their incomplete clinical data, 4 patients because of missing of their follow-up data and 15 patients because of their being younger than 18 years old. A total of 270 patients were enrolled into this study.

The duration of follow-up was 22 months (6-66 months) and 31 patients were defined as poor prognosis, of which 24 were dead. The baseline clinical data of two groups are shown in Table 1. HALP (44.46 \pm 32.97 vs. $29.68 \pm 21.80, P<0.001)$ was significantly higher in the good outcome group, which was clearly illustrated in Figure 1. In Table1, older patients were identified more frequently in the poor outcome group than in the good outcome group (33.36 \pm 12.49 vs. $42.00 \pm 14.84, P=0.002)$. Furthermore, coma was more common among patients with poor outcome $(P<0.001)$. As for laboratory parameters, lymphocyte reached statistical significance $(P<0.001)$. Additionally, gender $(P=0.044)$ was also regarded as a risk factor: female patients had poor outcome more regularly (14.1\%). Straight sinus was involved in the poor outcome group $(P=0.011)$.

HALP of $\geq 31.54$ and age of $\geq 36.5$ were found as threshold values by ROC curve. The discriminatory capability of HALP using the area under the ROC curve was 0.749 ( $95 \% \mathrm{Cl}: 0.633-0.865, P=0.044$ ), and HALP predicted the presence of good outcome with a sensitivity of $69.7 \%$ and specificity of $82.6 \%$ (Figure 2). As illustrated in Table 2, after adjusting for age, gender, coma and straight sinus which reached statistical significance in logistic regression, multivariable logistic regression analysis demonstrated that HALP (OR=0.978, 95\% Cl: 0.958-0.999, $P=0.039$ ) was a protective predictor of outcome. The weights and points associated with the five variables are shown in Figure 3 . The nomogram indicated that age $\geq 36.5$, 
female, coma and straight sinus thrombosis location were the poor prognostic factors, but higher HALP was still a potential good prognostic factor. The results of the nomogram were similar to those of aforementioned multivariate analyses in tumor ${ }^{10-12}$.

As seen in Figure 4, the decision curve analysis graphically showed the clinical usefulness of the model based on a continuum of potential thresholds for poor outcome risk (x axis) and the net benefit of using the model to risk stratify patients (y axis) relative to assuming that all patient will have a good prognosis. In this analysis, it demonstrated that this model significantly improved risk prediction at threshold probabilities of CVST at 0 to $85 \%$ compared with doing-none or doing-all. Kaplan-Meier analysis was used to determine the prognostic significance of HALP (Figure 5). Patients with higher HALP presented significantly higher overall survival rates $(P=0.006)$ than those with lower HALP.

\section{Discussion}

Our study mainly investigated the association between HALP and the prognosis in acute or subacute CVST patients and tried to provide a new model for clinical prognosis in patients. We found HALP to be a potential independent protective predictor of prognosis in acute and subacute CVST patients. Our new prognostic model with HALP had potentially better value for Chinese CVST patients.

Previous studies showed that a high HALP score predicted good therapeutic outcomes and prognosis in variety of tumors ${ }^{15-17}$, which strengthened the findings of our study. There has been more evidence about the relationship between these four single parameters and CVST. A systematic review and meta-analysis of the literature confirmed that elevated hemoglobin was associated with poorer outcomes in acute stroke, suggesting a U-shaped relationship between hemoglobin levels and stroke mortality ${ }^{18}$. Furthermore, in a case-control study including 152 CVST patients and 291 healthy controls, lower hemoglobin was a potential risk factor for $\mathrm{CVST}^{19}$. It can infer that moderate increase of hemoglobin may be beneficial to the prognosis of CVST patients. Serum albumin, the most abundant plasma protein, has many physiological properties, including anti-inflammatory, antioxidant and antiplatelet aggregation activity ${ }^{20,21}$. In a study that included 759 patients with acute ischemic stroke followed for 3 months, poor outcome was independently linked to low serum albumin level, ischemic heart disease and infarct size $(P<0.05 \text { for all })^{22}$. Large amount of evidence demonstrated that hypoalbuminemia is a strong, independent prognostic factor in stroke or $\mathrm{CVST}^{21-24}$. Brain ischemia is followed by the activation of the immune system in a sterile inflammatory reaction, resulting in the change of cell adhesion molecules, cytokines, as well as infiltration of leukocytes in the ischemic tissue. Lymphocytes, as an important subtype of the leukocyte family, are also involved in inflammation. Previous studies have demonstrated the association between the low lymphocyte count and increased cardiovascular disease ${ }^{25}$. It was discovered in an animal model that stroke-induced immunosuppression could result in lymphopenia ${ }^{26}$. In human studies, lymphopenia deactivation was also observed in peripheral blood after stroke, especially during its acute phase ${ }^{27}$. Furthermore, some trials have shown an increased association between platelets and development of vascular events 28,29 . The basis of sinus venous thrombosis can be linked 
to Virchow's triad, which includes injury to the vessel walls, a hypercoagulable state and stasis ${ }^{30}$. Platelets are involved in early stage of vascular pathology, which inside the plaque ensure replication of leukocytes via direct receptor interactions and increase the leukocyte activity ${ }^{31}$. According to the heat map in Figure 1, it was clear that patients with poor outcome had lower HALP than those with good outcome. But the four single parameters did not show obvious trends. This was one of the reasons that we made the definition of HALP. The results of our study confirmed the significance of HALP, indicating that the patients with higher HALP had better prognosis. Nevertheless, we still required more randomized controlled trials to strengthen the potential benefits.

Such factors, as PLR, NLR, LMR, D-dimer, C-reactive protein, red cell distribution width and mean platelet volume, provided important information regarding the prognosis of CVST patients ${ }^{5,32}$. In addition, the previously validated risk score derived from the ISCVT study and the VENOPORT registry (the ISCVT-RS system) has been most commonly accepted ${ }^{6}$, including gender, mental disorder, coma, venous thrombosis, intracerebral hemorrhage and malignancy. But more and more evidences showed that ISCVTRS scores have some limitations. A Chinese retrospective study was conducted with this scale. It is found that the accuracy is not ideal, and the AUC is $0.65(95 \% \mathrm{Cl} 0.53-0.77, P<0.01)^{8}$. It may be due to the heterogeneity of the research population ${ }^{33}$. Our previous studies have similar findings. And we have reasons to believe that this scale needs to be further updated and improved, especially for the Chinese population. Therefore, we evaluated HALP and explored prognosis models which are suitable for our Chinese patients. The DCA was used to analyze the benefit of prognostic model commonly used in clinical practice in Figure 4. In this analysis, the red line is almost 0 to 1 above the gray line and the black line, and we could think that the prognostic model can benefit more patients.

In our study, to improve the reliability, we figured out the nomogram to visually show the impact of some clinic-pathological parameters on the prognosis of CVST patients. In this nomogram, HALP was included via a stepwise algorithm. According to the nomogram, the prognosis of individual patient could be well predicted. We also noticed HALP and age might play a similarly important role in prognosis, which was a very exciting result.

There were several limitations in our study. First, the study was a single-center study and selection bias was unavoidable. Additional well-designed and larger prospective cohort multicenter studies are required to evaluate this association. Second, HALP was only collected once, thus there was a lack of dynamic data. Further evidence is required to evaluate potential values.

\section{Conclusion}

Our findings suggested that higher HALP value may indicate an independent predictor for good prognosis in acute and subacute CVST patients. The new prognostic model with HALP had potentially better value for Chinese CVST patients.

\section{Abbreviations}


CVST: Cerebral Venous Sinus Thrombosis; ISCVT: International Study on Cerebral Vein and Dural Sinus Thrombosis; ISCVT-RS: CVST prognostic score based on the International Study on Cerebral Vein and Dural Sinus Thrombosis; CVT-GS: Cerebral Venous Thrombosis grading scale; HALP hemoglobin $(\mathrm{g} / \mathrm{L}) \times$ albumin (g/L) x lymphocyte (/L)/platelet (/L); PLR: Platelet to Lymphocyte Ratio; NLR: Neutrophil to Lymphocyte Ratio; mRS: modified Rankin Scale; AUC: Area under the ROC curve; Cl: Confidence Interval; ROC: Receiver Operating Characteristic; OR: Odds Ratio; VENOPORT: Cerebral Venous Thrombosis Portuguese Collaborative Study; ISCVT-V: Research and Validation sample for ISCVT.

\section{Declarations}

\section{Ethics approval and consent to participate}

The retrospective cohort study was approved by the Ethics Committee of the First Affiliated Hospital of Zhengzhou University. The requirement for written informed consent was waived due to its retrospective nature.

\section{Consent for publication}

Not applicable

\section{Availability of data and materials}

The data is not available because of patients' privacy.

\section{Competing interests}

The authors declare that they have no competing interests.

\section{Funding}

This work was supported by the Science and Technology Department of the Henan Province grant number (No.152102310058), the National Natural Science Foundation of China grants (Nos. 81530037, 31970158).

\section{Authors' contributions}

Shen $\mathrm{Li}$ is the first author. Dr. Bo Song, Dr. Yuming Xu and Dr. Zongping Xia provided funding and designed the study. Dr. Bo Song and Dr. Zongping Xia revised the total article. Dr. Hongbing Liu, Dr. Jiawei Zhao and Dr. Hui Fang collected the data. Dr. Kai Liu, Dr. Yuan Gao, Dr. Lu Zhao and Dr. Zhang Rui were involved in data cleaning, follow-up, and verification. All authors have read and approved the final manuscript.

\section{Acknowledgements}


We thank Dr. Chunjie Zhang (Department of Medical record, school of the First Affiliated Hospital of Zhengzhou University) for his technical consulting service.

\section{References}

1. Sader N, de Lotbiniere-Bassett M, Tso MK, Hamilton M. Management of venous sinus thrombosis. Neurosurg Clin North Am. 2018;29:585-94.

2. Coutinho JM, Zuurbier SM, Aramideh M, Stam J. The incidence of cerebral venous thrombosis: A cross-sectional study. Stroke. 2012;43:3375-7.

3. Akboga YE, Bektas H, Anlar O. Usefulness of platelet to lymphocyte and neutrophil to lymphocyte ratios in predicting the presence of cerebral venous sinus thrombosis and in-hospital major adverse cerebral events. Journal of the neurological sciences. 2017;380:226-9.

4. Artoni A, Abbattista M, Bucciarelli P, Gianniello F, Scalambrino E, Pappalardo E, et al. Platelet to lymphocyte ratio and neutrophil to lymphocyte ratio as risk factors for venous thrombosis. Clinical applied thrombosis/hemostasis: official journal of the International Academy of Clinical Applied Thrombosis/Hemostasis. 2018;24:808-14.

5. Meng R, Wang X, Hussain M, Dornbos D 3rd, Meng L, Liu Y, et al. Evaluation of plasma d-dimer plus fibrinogen in predicting acute cvst. International journal of stroke: official journal of the International Stroke Society. 2014;9:166-73.

6. Ferro JM, Bacelar-Nicolau H, Rodrigues T, Bacelar-Nicolau L, Canhao P, Crassard I, et al. Risk score to predict the outcome of patients with cerebral vein and dural sinus thrombosis. Cerebrovascular diseases. 2009;28:39-44.

7. Barboza MA, Chiquete E, Arauz A, Merlos-Benitez M, Quiroz-Compean A, Barinagarrementeria F, et al. A practical score for prediction of outcome after cerebral venous thrombosis. Front Neurol. 2018;9:882.

8. Li S, Liu K, Zhang R, Gao Y, Fang H, Liu X, et al. Lower lymphocyte tomonocyte ratio is a potential predictor of poor outcome in patients with cerebral venous sinus thrombosis. Stroke Vasc Neurol. 2019 Sep;4(3).

9. Chen XL, Xue L, Wang W, Chen HN, Zhang WH, Liu K, et al. Prognostic significance of the combination of preoperative hemoglobin, albumin, lymphocyte and platelet in patients with gastric carcinoma: a retrospective cohort study. Oncotarget. 2015 Dec 01;6(38).

10. Peng D, Zhang CJ, Gong YQ, Hao H, Guan B, Li XS, et al. Prognostic significance of halp (hemoglobin, albumin, lymphocyte and platelet) in patients with bladder cancer after radical cystectomy. Scientific reports. 2018;8:794.

11. Cong $L, H u L$. The value of the combination of hemoglobin, albumin, lymphocyte and platelet in predicting platinum-based chemoradiotherapy response in male patients with esophageal squamous cell carcinoma. Int Immunopharmacol. 2017;46:75-9. 
12. Peng D, Zhang CJ, Tang Q, Zhang L, Yang KW, Yu XT, et al. Prognostic significance of the combination of preoperative hemoglobin and albumin levels and lymphocyte and platelet counts (halp) in patients with renal cell carcinoma after nephrectomy. BMC Urol. 2018;18:20.

13. Wang HF, Pu CQ, Yin X, Tian CL, Chen T, Guo JH, et al. D-dimers (DD) in CVST. International Journal of Neuroscience. 2017 Jun;127(6).

14. De la Vega Muns G, Quencer R, Ezuddin NS, Saigal G. Utility of Hounsfield unit and hematocrit values in the diagnosis of acute venous sinus thrombosis in unenhanced brain CTs in the pediatric population. Pediatric Radiology. 2019 02;49(2).

15. Jurasz P, Alonso-Escolano D, Radomski MW. Platelet-cancer interactions: Mechanisms and pharmacology of tumour cell-induced platelet aggregation. Br J Pharmacol. 2004;143:819-26.

16. Mantovani A, Allavena P, Sica A, Balkwill F. Cancer-related inflammation. Nature. 2008;454:436-44.

17. Jiang N, Deng J-Y, Liu Y, Ke B, Liu H-G, Liang H. The role of preoperative neutrophil-lymphocyte and platelet-lymphocyte ratio in patients after radical resection for gastric cancer. Biomarkers. 2014;19:444-51.

18. Barlas RS, Honney K, Loke YK, McCall SJ, Bettencourt-Silva JH, Clark AB, et al. Impact of hemoglobin levels and anemia on mortality in acute stroke: Analysis of uk regional registry data, systematic review, and meta-analysis. Journal of the American Heart Association. 2016;5.

19. Coutinho JM, Zuurbier SM, Gaartman AE, Dikstaal AA, Stam J, Middeldorp S, et al. Association between anemia and cerebral venous thrombosis: Case-control study. Stroke. 2015;46:2735-40.

20. Lam FW, Cruz MA, Leung HC, Parikh KS, Smith CW, Rumbaut RE. Histone induced platelet aggregation is inhibited by normal albumin. Thrombosis research. 2013;132:69-76.

21. Zhang Q, Lei YX, Wang Q, Jin YP, Fu RL, Geng HH, et al. Serum albumin level is associated with the recurrence of acute ischemic stroke. Am J Emerg Med. 2016;34:1812-6.

22. Famakin B, Weiss P, Hertzberg V, McClellan W, Presley R, Krompf K, et al. Hypoalbuminemia predicts acute stroke mortality: Paul coverdell georgia stroke registry. Journal of stroke cerebrovascular diseases: the official journal of National Stroke Association. 2010;19:17-22.

23. Dziedzic T, Slowik A, Szczudlik A. Serum albumin level as a predictor of ischemic stroke outcome. Stroke. 2004;35:e156-8.

24. Idicula TT, Waje-Andreassen U, Brogger J, Naess H, Thomassen L. Serum albumin in ischemic stroke patients: The higher the better. The bergen stroke study. Cerebrovascular diseases. 2009;28:13-7.

25. Horne BD, Anderson JL, John JM, Weaver A, Bair TL, Jensen KR, et al. Which white blood cell subtypes predict increased cardiovascular risk? J Am Coll Cardiol. 2005;45:1638-43.

26. Prass K, Meisel C, Hoflich C, Braun J, Halle E, Wolf T, et al. Stroke-induced immunodeficiency promotes spontaneous bacterial infections and is mediated by sympathetic activation reversal by poststroke t helper cell type 1-like immunostimulation. The Journal of experimental medicine. 2003;198:725-36. 
27. Vogelgesang A, Grunwald U, Langner Sn, Jack R, Bröker BM, Kessler C, et al. Analysis of lymphocyte subsets in patients with stroke and their influence on infection after stroke. Stroke. 2008;39:237-41.

28. Oz II, Yucel M, Bilici M, Serifoglu I, Sayin R, Ilikhan SU, et al. Is mean platelet volume a reliable marker to predict ischemic stroke in the follow-up of patients with carotid stenosis? Journal of stroke cerebrovascular diseases: the official journal of National Stroke Association. 2016;25:404-9.

29. Arevalo-Lorido JC, Carretero-Gomez J, Alvarez-Oliva A, Gutierrez-Montano C, Fernandez-Recio JM, Najarro-Diez F. Mean platelet volume in acute phase of ischemic stroke, as predictor of mortality and functional outcome after 1 year. Journal of stroke cerebrovascular diseases: the official journal of National Stroke Association. 2013;22:297-303.

30. Li S, Liu K, Gao Y, Zhao L, Zhang R, Fang H, et al. Prognostic value of systemic immuneinflammation index in acute/subacute patients with cerebral venous sinus thrombosis. Stroke and Vascular Neurology. 2020:svn-2020-000362.

31. Gawaz M, Langer H, May AE. Platelets in inflammation and atherogenesis. J Clin Investig. $2005 ; 115: 3378-84$.

32. Talbot $K$, Wright M, Keeling D. Normal d-dimer levels do not exclude the diagnosis of cerebral venous sinus thrombosis. Journal of neurology. 2002;249:1603-4.

33. Koopman K, Uyttenboogaart M, Vroomen PC, van der Meer J, De Keyser J, Luijckx GJ. Development and validation of a predictive outcome score of cerebral venous thrombosis. Journal of the neurological sciences. 2009;276:66-8.

\section{Tables}

Table 1. Demographic and clinical characteristics of the two outcomes groups in CVST patients 


\begin{tabular}{|c|c|c|c|c|}
\hline Variable & Total & Good(239, 89.0\%) & Poor(31,11.0 \%) & $P$ value \\
\hline Age & $34.50 \pm 13.12$ & $33.36 \pm 12.49$ & $42.00 \pm 14.84$ & $0.002^{*}$ \\
\hline Gender & 270 & 239 & 31 & $0.044^{*}$ \\
\hline Female & 156 & 134 & 22 & \\
\hline Male & 114 & 105 & 9 & \\
\hline Malignancy & 4 & 3 & 1 & 0.575 \\
\hline Infection & 57 & 48 & 9 & 0.511 \\
\hline Pregnancy or Puerperium & 65 & 53 & 12 & 0.518 \\
\hline Intracerebral hemorrhage & 62 & 53 & 9 & 0.674 \\
\hline Coma & 72 & 50 & 22 & $<0.001 *$ \\
\hline Mental disorder & 15 & 10 & 5 & 0.303 \\
\hline Lymphocyte & $1.67 \pm 0.84$ & $1.74 \pm 0.85$ & $1.19 \pm 0.62$ & $<0.001 *$ \\
\hline Granulocyte & $7.27 \pm 4.73$ & $7.04 \pm 4.63$ & $8.79 \pm 5.15$ & 0.066 \\
\hline Platelet & $244.12 \pm 124.21$ & $243.15 \pm 122.08$ & $250.54 \pm 139.23$ & 0.768 \\
\hline Albumin & $40.31 \pm 5.23$ & $40.41 \pm 5.34$ & $39.64 \pm 4.46$ & 0.358 \\
\hline Hemoglobin & $126.96 \pm 27.51$ & $127.51 \pm 27.77$ & $123.34 \pm 25.89$ & 0.384 \\
\hline HALP & $42.51 \pm 32.09$ & $44.46 \pm 32.97$ & $29.68 \pm 21.80$ & $0.001 *$ \\
\hline PLR & $179.47 \pm 129.72$ & $159.61 \pm 90.38$ & $339.32 \pm 257.80$ & $<0.001 *$ \\
\hline NLR & $6.81 \pm 7.90$ & $5.50 \pm 5.15$ & $17.05 \pm 15.94$ & $<0.001 *$ \\
\hline Left sigmoid sinus & 70 & 47 & 23 & 0.629 \\
\hline Right sigmoid sinus & 75 & 57 & 18 & 0.218 \\
\hline Left transverse sinus & 98 & 63 & 35 & 0.147 \\
\hline Right transverse sinus & 97 & 73 & 24 & 0.188 \\
\hline Straight sinus & 21 & 14 & 7 & $0.011 *$ \\
\hline Superior sagittal sinus & 159 & 119 & 40 & 0.243 \\
\hline Inferior sagittal sinus & 25 & 13 & 12 & 0.061 \\
\hline Torcular & 28 & 18 & 10 & 0.510 \\
\hline Deep cerebral venous & 8 & 6 & 2 & 0.284 \\
\hline
\end{tabular}

*Statistically significant 
Table 2. Multivariable logistic regression analysis in CVST patients

\begin{tabular}{|llll|}
\hline Variable & OR & $95 \% \mathrm{Cl}$ & P value \\
\hline Age & 1.054 & $1.022-1.086$ & $0.001^{*}$ \\
\hline Gender & 3.321 & $0.704-6.661$ & 0.129 \\
\hline Coma & 5.440 & $2.344-8.624$ & $0.001^{*}$ \\
\hline HALP & 0.978 & $0.901-0.989$ & $0.03{ }^{*}$ \\
\hline Straight sinus & 5.683 & $1.702-8.980$ & $0.005^{*}$ \\
\hline
\end{tabular}

*Statistically significant

\section{Figures}

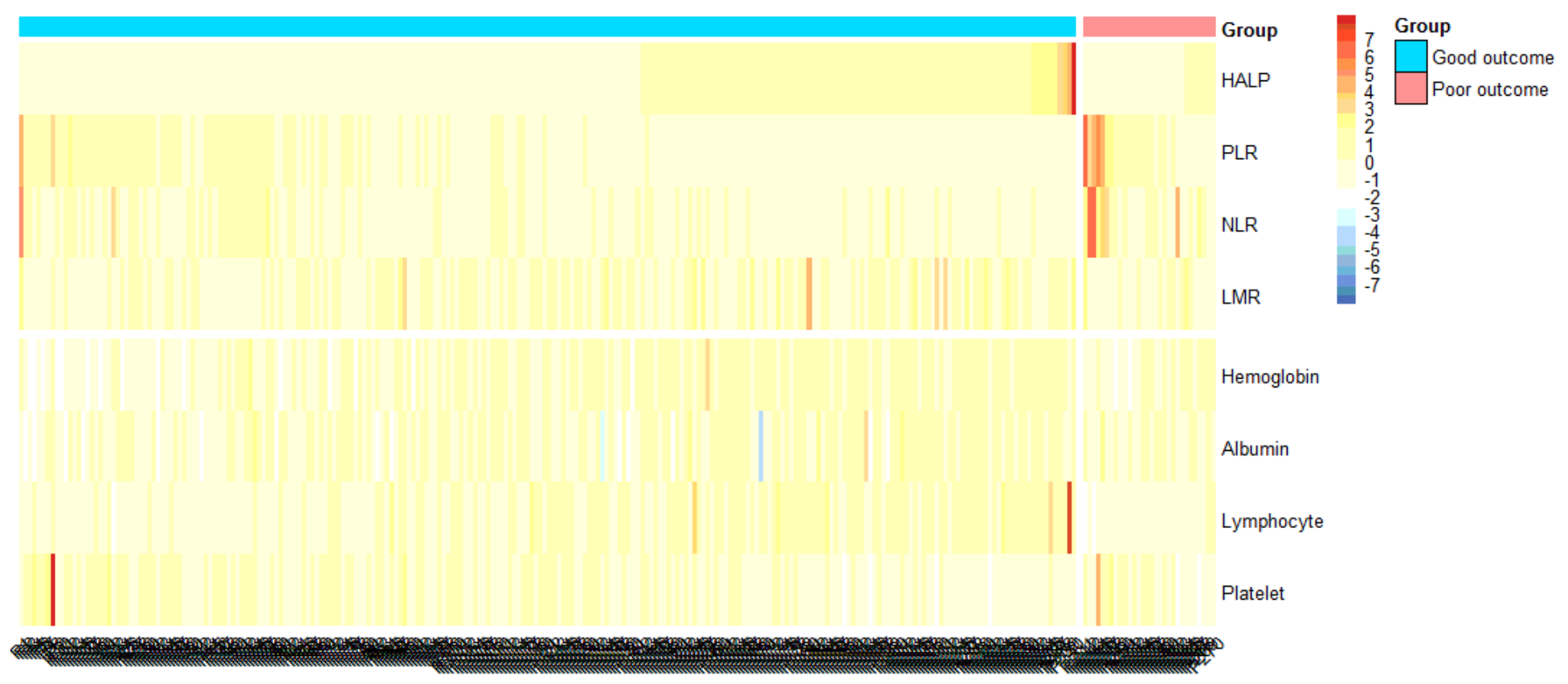

Figure 1

Heat map identifying potential prognostic values in CVST patients. 




Figure 2

ROC curve of HALP in CVST patients.

Points

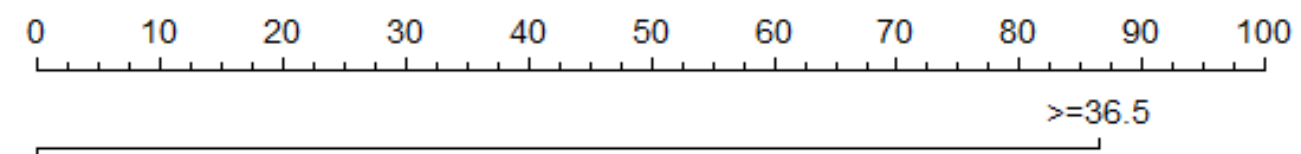

Age

Gender

Coma

SS

HALP

Total Points

Risk of hypertension

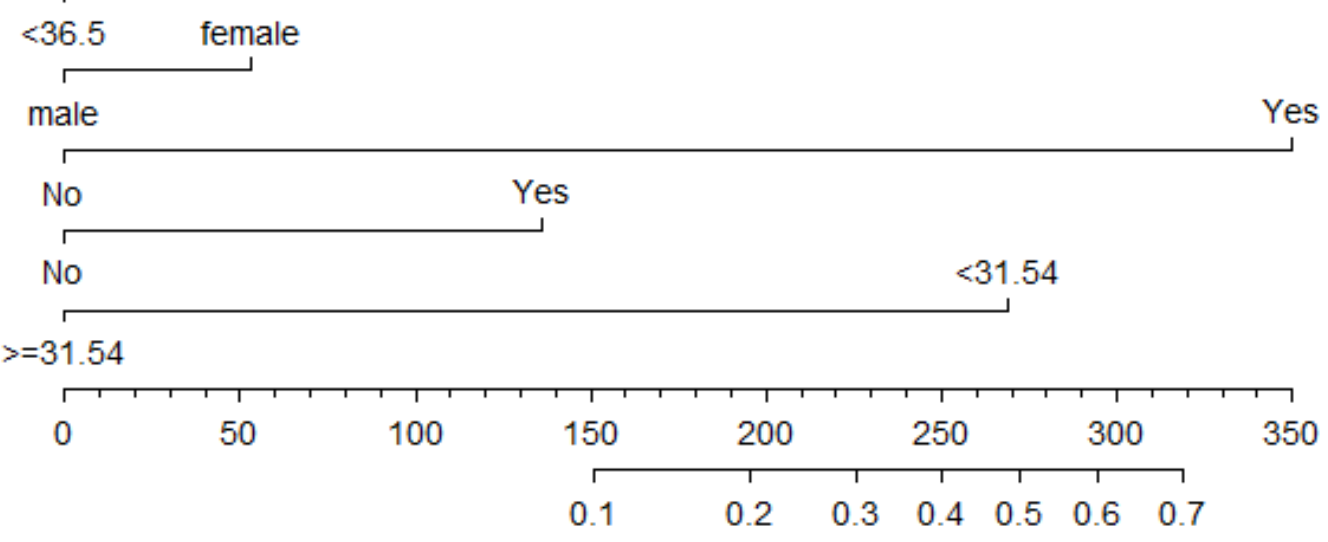

Figure 3

Nomogram for the new values-based risk score in CVST patients. 


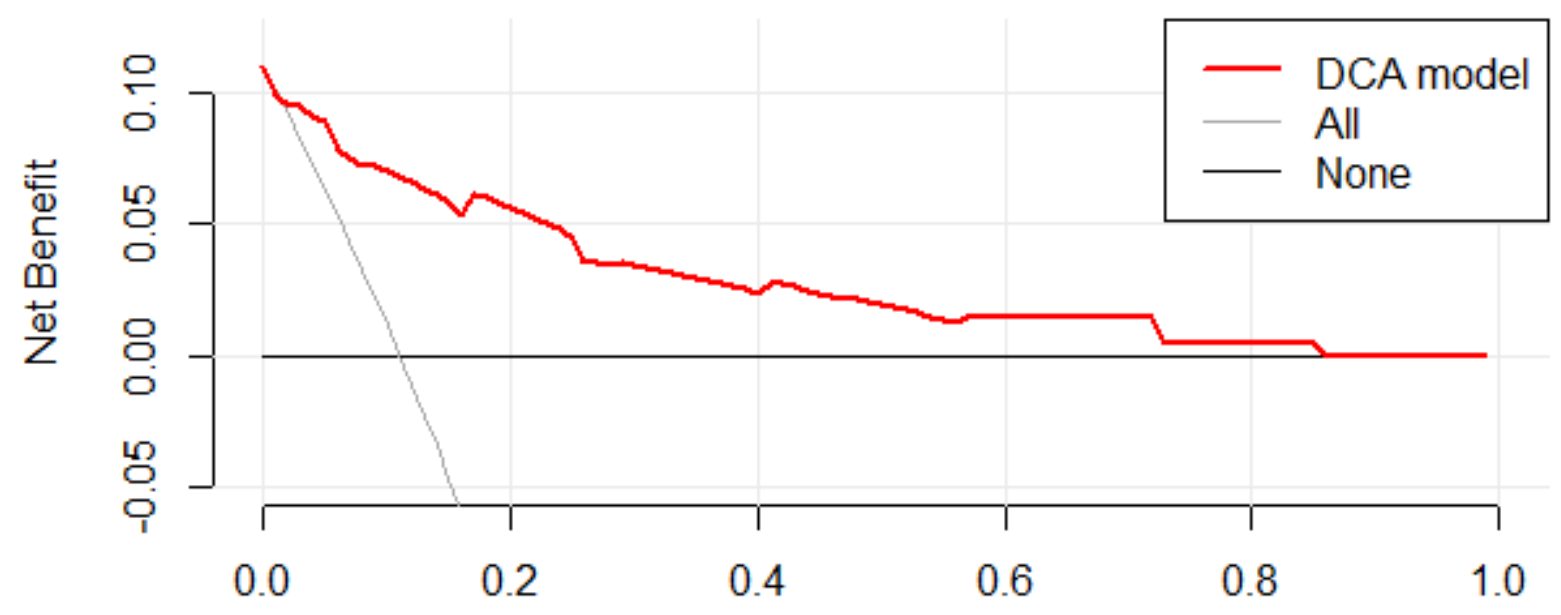

Threshold probability

Figure 4

Decision curve analysis for the novel prediction model of CVST patients.

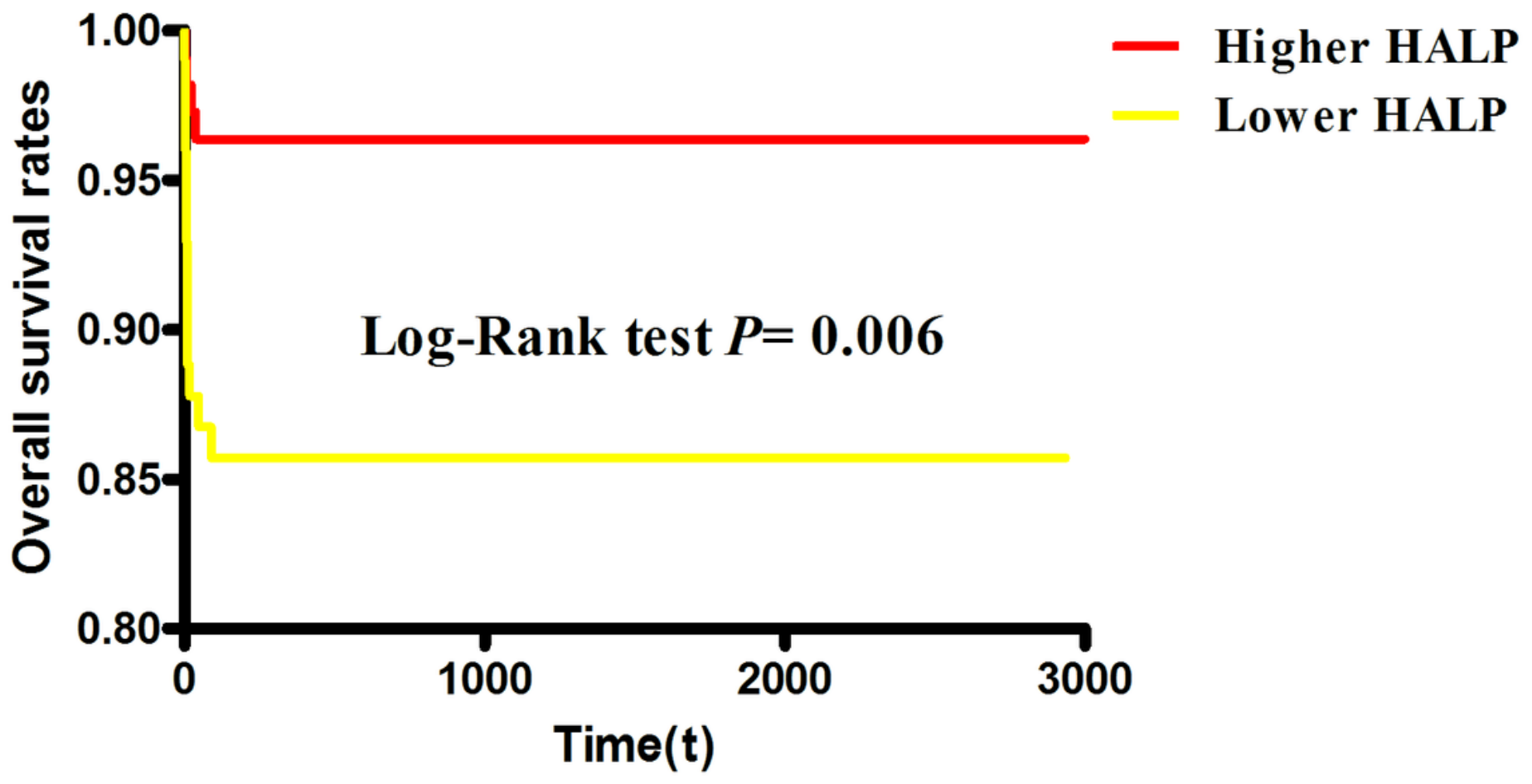

Figure 5

Kaplan Meier analysis for CVST patients with high/low HALP. patients. 